\title{
First principles prediction of structural and electronic properties of $\mathrm{Tl}_{x} \operatorname{In}_{1-x} \mathrm{~N}$ alloy
}

\author{
M. J. Winiarski ${ }^{\mathrm{a}}$, P. Scharoch ${ }^{\mathrm{b}}$, M. P. Polak ${ }^{\mathrm{b}}$ \\ ${ }^{a}$ Institute of Low Temperature and Structure Research, Polish Academy of Sciences, \\ Okólna 2, 50-422 Wroctaw, Poland, EU \\ ${ }^{b}$ Institute of Physics, Wroclaw University of Technology, Wyb. Wyspianskiego 27, 50-370 \\ Wroclaw, Poland, EU
}

\begin{abstract}
Structural and electronic properties of zinc blende $\mathrm{Tl}_{x} \operatorname{In}_{1-x} \mathrm{~N}$ alloy have been evaluated from first principles. The band structures have been obtained within the density functional theory (DFT), the modified Becke-Johnson (MBJLDA) approach for the exchange-correlation potential, and fully relativistic pseudopotentials. The calculated band-gap dependence on Tl content in this hypothetical alloy exhibits a linear behaviour up to the $25 \%$ of thalium content where its values become close to zero. In turn, the split-off energy at the $\Gamma$ point of the Brillouin zone, related to the spin-orbit coupling, is predicted to be comparable in value with the band-gap for relatively low thalium contents of about $5 \%$. These findings suggest $\mathrm{Tl}_{x} \operatorname{In}_{1-x} \mathrm{~N}$ alloy as a promising material for optoelectronic applications. Furthermore, the band structure of TIN reveals some specific properties exhibited by topological insulators.
\end{abstract}

\section{Introduction}

Group III metal nitrides have been widely explored materials for optoelectronic applications. Forming of ternary and quaternary alloys in the wurtzite phase offers a promising perspective of tuning their direct band-gaps starting from 0.65-0.69 eV [1, 2, 3] for InN, through 3.50-3.51 eV [4, 5] for GaN, up to $6.1 \mathrm{eV}$ for AlN [5], which is crucial for optoelectronics. Especially the In-based systems have been extensively investigated due to their small band-gaps [6, 7, 8, 9].

Optical properties of some thalium III-V alloys have already been reported. First, the theoretical investigations of $\mathrm{Tl}_{x} \mathrm{In}_{1-x} \mathrm{Sb}[10]$ and $\mathrm{Tl}_{1-x} \operatorname{In}_{x} \mathrm{P}$ 
[11] suggested these materials as potential candidates for infrared detectors. Next, experimental studies extended this idea on ternary $\mathrm{Tl}_{1-x} \operatorname{In}_{x} \mathrm{P}$ [12], $\mathrm{Tl}_{1-x} \mathrm{Ga}_{x} \mathrm{As}$, and $\mathrm{Tl}_{1-x} \mathrm{In}_{x}$ As alloys [13, 14].

Although the synthesis of thalium nitride has not been reported yet, this compound has been studied theoretically [15, 16, 17] because of the predicted existence of semimetallic phase, i.e. the situation where creating an alloy by adding a wide band-gap component and controlling its content may cover a very broad range of band-gaps. The structural and elastic properties of TIN, as technologically important, have been investigated in equilibrium conditions and under hydrostatic pressure [18, 19]. The electronic and structural properties of $\mathrm{Tl}_{x} \mathrm{Al}_{1-x} \mathrm{~N}$ [20, 21] and $\mathrm{Tl}_{x} \mathrm{Ga}_{1-x} \mathrm{~N}$ [22] have been also studied $a b$ initio, however, these investigations were performed with the standard DFT exchange-correlation functionals.

The standard (LDA/GGA) DFT calculations lead to unrealistic band structures for narrow band-gap semiconductors, and as a result, to false conclusions about the real value of the band-gap as well as the spin-orbit coupling (SOC) driven separation between the heavy hole and the split-off band at the $\Gamma$ point of the Brillouin zone. Thus, such technologically important properties of semiconducting alloys as band-gap bowing and the split-off energy $\left(\Delta_{S O}\right)$ cannot be studied within LDA/GGA approach.

In this work, the modified Becke-Johnson potential (MBJLDA) proposed by Tran and Blaha [23] was used for the band structure calculations. The performance of this method has been already tested for small band-gap systems, eg. InAs and InSb [24], thus the results for zinc blende $\mathrm{Tl}_{x} \operatorname{In}_{1-x} \mathrm{~N}$ alloy reported here may be considered as a reliable prediction of the band structure properties of this hypothetical novel alloy.

\section{Computational details}

The electronic structure calculations for zinc blende (ZB) $\mathrm{Tl}_{x} \operatorname{In}_{1-x} \mathrm{~N}$ alloy have been performed with the use of the ABINIT code [25, 26]. First, the equilibrium geometries were found via stresses/forces relaxation for PAW atomic datasets generated with the Atompaw package [27] with the PerdewWang [28] parametrization for the exchange-correlation energy. This was followed by the calculations of the band structures with the use of the normconserving fully relativistic pseudopotentials generated using the Atomic Pseudopotential Engine [29]. The MBJLDA (TB09) [23] functional, which consists of the modified Becke-Johnson exchange potential combined with 
an LDA correlation, was used. This complex calculation scheme was necessary to obtain reasonable results of LDA predicted structural parameters and MBJLDA band-gaps with spin-orbit coupling effects included. A supercell consisting of 16 -atoms $(2 \times 2 \times 2$ multiplicity of primitive FCC cell $)$ was used to simulate the alloy. The most possibly uniform arrangements of atoms were chosen. The total energy convergence in the plane wave basis was found to be sufficient for $30 \mathrm{Ha}$ energy cutoff and the $4 \times 4 \times 4$ k-point mesh with standard shifts for FCC lattice.

It should be noted that calculations in the pseudopotential approach lead to an underestimation of the $c$ parameter in the MBJLDA exchangecorrelation functional [23], and as a result, to a relatively low value of $\mathrm{InN}$ band-gap. In this work, $c=1.335$ was chosen to obtain the band-gap value of $0.69 \mathrm{eV}$, the same as computed with the full-potential MBJLDA calculations

with the Wien2k code [30]. An analogous discussion has been also contained in ref. [31].

\section{Results and discussion}

Equilibrium lattice parameters, split-off energies and band-gaps calculated for InN and TIN are gathered in Tab.1. Although the (LDA) lattice parameter obtained here for $\mathrm{TIN}$ is in a good agreement with the literature data, the $a=4.948 \AA$ for $\mathrm{InN}$ is significantly higher than the value of 4.800 $\AA$ [32] reported earlier, and slightly different than our former result [31] from pseudopotential calculations. It is worth to note that the PAW atomic data used here contained the full set of valence states, including the semicore In $4 \mathrm{~s}$ and $4 \mathrm{p}$ states, thus the presented here structural parameters seem to be the most adequate DFT-derived values.

The MBJLDA band-gap value for $\mathrm{ZB} \mathrm{InN}, \mathrm{E}_{g}=0.69 \mathrm{eV}$, computed with the full-potential method (Wien2k), being reasonable compared to literature, was used as a reference value in fully relativistic pseudopotential calculations performed in this work. Interestingly, both the fully relativistic LDA and MBJLDA approaches gave a nonzero direct band-gap for TIN in the $\Gamma$ point, in contrast to former LDA/GGA investigations without SOC [22], however significantly smaller than $0.050 \mathrm{eV}$ reported for fully relativistic GGA calculations [20].

The $\Delta_{S O}$ obtained with the LDA approach for InN is strongly overestimated. Since LDA calculations lead to artificially negative band-gap of InN, 
the position of the split-off band is not related to the SOC effects. The MBJLDA calculations provide more proper description of InN band structure, presented in Fig. 1, In this case, the real SOC-driven $\Delta_{S O}=0.033 \mathrm{eV}$ is an order of magnitude lower than $0.393 \mathrm{eV}$ obtained with LDA, thus hardly visible in the scale of Fig. 1, whereas some distinct splittings of heavy and light-hole bands can be seen in the $X$ point as well as along $X-\Gamma$ direction in the BZ of InN.

One can consider an analogous issue for TIN, despite that in this compound the SOC effect could have been expected to be very strong. In this case the MBJLDA calculations also lead to a significant reduction of $\Delta_{S O}$ from $2.083 \mathrm{eV}$ obtained with LDA to a more reasonable value of $1.397 \mathrm{eV}$. The band structure of TIN is depicted in Fig. 2. The strong SOC effects are clearly visible in all directions in TIN band structure. A former study reported a band-gap inversion in this system [22], which is also present in the fully relativistic MBJLDA results, as can be seen in band character plot presented in Fig. 3.

Interestingly, both LDA and MBJLDA approaches suggest a narrow and somewhat indirect band-gap in the vicinty of the $\Gamma$ point in BZ of TIN. This effect is related to the anomalous order of $\mathrm{N} 2 \mathrm{p}$ bands, since the quartet $\mathrm{N}$ $2 \mathrm{p}_{3 / 2}$ is below the doublet $\mathrm{N} 2 \mathrm{p}_{1 / 2}$. Analogous properties of band structure have been revealed for $\beta$-HgS which is suggested to be a strong 3D topological insulator [34], thus TIN may also be considered as a candidate compound for a topological insulator. Despite that the parity criteria [35] do not apply to the binary ZB-type structure due to the lack of inversion symmetry, the simple predictions based only on a topological band-order are generaly accepted in literature, e.g. for InSb [36]. Therefore, in this work TIN is expected to exhibit a similar surface state behaviour to that of $\beta$-HgS, although the surface calculations for TIN have not been performed.

The lattice parameter of $\mathrm{ZB} \mathrm{Tl}_{x} \operatorname{In}_{1-x} \mathrm{~N}$ alloy reveals Vegard's law behaviour, as depicted in Fig 4. A similar linear dependence of lattice parameters of an alloy was reported for $\mathrm{Tl}_{x} \mathrm{Al}_{1-x} \mathrm{~N}$ [20], which may suggest that the deviation from Vegard's law for $\mathrm{Tl}_{x} \mathrm{Ga}_{1-x} \mathrm{~N}$ in ref. [22] needs a careful verification.

The band-gaps $\left(\mathrm{E}_{g}\right)$, and the split-off energies $\left(\Delta_{S O}\right)$ of $\mathrm{Tl}_{x} \operatorname{In}_{1-x} \mathrm{~N}$ alloy are depicted in Fig,5. The dependence of the alloy band-gap as a function of $\mathrm{Tl}$ content predicted here is somewhat unusual compared to those of other group III-V nitride alloys. However, a similar behaviour has also been reported for $\mathrm{Tl}_{x} \mathrm{Al}_{1-x} \mathrm{~N}$ for a relatively high thalium contents [21]. This effect 
is related to the semimetallic character of TIN and its strongly ionic bonding [15], on the contrary to semiconducting systems. Thus, the introduction of $\mathrm{Tl}$ atoms into InN leads to a strong modification of electronic structure.

The calculated $\Delta_{S O}$ for $\mathrm{Tl}_{x} \operatorname{In}_{1-x} \mathrm{~N}$ alloy, presented in Fig. 5 , indicates a very strong enhancement of SOC introduced by $\mathrm{Tl}$ atoms. Since InN is a small band-gap system, the predicted value of $\Delta_{S O}$ in $\mathrm{Tl}_{x} \operatorname{In}_{1-x} \mathrm{~N}$ alloy is comparable to the band-gap for a relatively low thalium contents, about $5 \%$. The $\Delta_{S O}$ is an important parameter for optoelectronic devices since it is connected with the unwanted non-radiative Auger recombination 37] (CHSH transition), which is suppressed when $\Delta_{S O}$ is greater in value than $\mathrm{E}_{g}$. E.g. in the Bi-dopped GaAs this situation takes place for Bi content greater than 9-10\% [39]. Therefore, the presented here results for $\mathrm{Tl}_{x} \operatorname{In}_{1-x} \mathrm{~N}$ alloy indicate that this system is a promising material for applications in optoelectronics, even for a relatively low thalium contents. However, despite the successful synthesis of $\mathrm{Tl}_{1-x} \mathrm{In}_{x} \mathrm{P}$ [12], $\mathrm{Tl}_{1-x} \mathrm{Ga}_{x} \mathrm{As}$, and $\mathrm{Tl}_{1-x} \mathrm{In}_{x}$ As alloys [13, 14] there are still no reports on experimental data for any Tl-dopped group III nitrides.

In turn, thalium-dopped InN systems may be considered as candidate compounds for topological insulators with relatively wide band-gaps, thus the topological band-order in $\mathrm{Tl}_{x} \mathrm{In}_{1-x} \mathrm{~N}$ alloy seems to be an interesting topic for further investigations.

\section{Conclusions}

The fully relativistic MBJLDA investigations of electronic structure of $\mathrm{Tl}_{x} \operatorname{In}_{1-x} \mathrm{~N}$ alloy predict a linear decrease in alloy band-gap with increasing thalium content, as well as the semimetallic character above $x \approx 0.25$. The high split-off energy in the $\Gamma$ point of the BZ, introduced by spin-orbit coupling, indicates that this system is a promising material for optoelectronic devices. Futhermore, the $\mathrm{Tl}_{x} \operatorname{In}_{1-x} \mathrm{~N}$ alloy may also exhibit a topological insulator behaviour. The results presented in this work should encourage further theoretical and experimental studies of Tl-dopped group III nitride alloys.

\section{Acknowledgments}

The calculations were performed in Wrocław Centre for Networking and Supercomputing. 


\section{References}

[1] V. Y. Davydov, Phys. Stat. Sol. B 229 (2002) R1.

[2] S. X. Li, J. Wu, E. E. Haller, W. Walukiewicz, W. Shan, H. Lu, W. J. Schaff, Appl. Phys. Lett. 83 (2003) 4963.

[3] J. Wu, W. Walukiewicz, W. Shan, K. M. Yu, J. W. Ager, S. X. Li, E. E. Haller, H. Lu, W. J. Schaff, J. Appl. Phys. 94 (2003) 4457.

[4] Y. C. Yeo, T. C. Chong, M. F. Li, J. Appl. Phys. 83 (1998) 1429.

[5] I. I. Vurgaftman, J. R. Meyer, J. Appl. Phys. 94 (2003) 3675.

[6] I. Gorczyca, S. P. Lepkowski, T. Suski, N. E. Christensen, A. Svane, Phys. Rev. B 80 (2009) 075202.

[7] E. Sakalauskas, O. Tuna, A. Kraus, H. Bremers, U. Rossow, C. Giesen, M. Heuken, A. Hangleiter, G. Gobsch, R Goldhahn, Phys. Stat. Sol. B 249 (2012) 485.

[8] P. G. Moses, C. G. Van de Walle, Appl. Phys. Lett. 96 (2010) 021208.

[9] S. Zhang, J. Shi, S. Zhu, F. Wang, M. Yang, Z. Bao, Phys. Lett. A 374 (2010) 4767.

[10] M. Van Schilfgaarde, A. Sher, A. B. Chen, Appl. Phys. Lett. 62 (1993) 1857.

[11] M. Van Schilfgaarde, A. B. Chen, S. Krishnamurthy, A. Sher, Appl. Phys. Lett. 65 (1994) 2714.

[12] K. Yamamoto, H. Asahi, M. Fushida, K. Iwata, S. Gonda, J. Appl.Phys. 81 (1997) 1704.

[13] R. Beneyton, G. Grenet, Ph. Regreny, M. Gendry, G. Hollinger, B. Canut, C. Priester, Phys. Rev. B 72 (2005) 125209.

[14] Y. Kajikawa, H. Kubota, S. Asahina, N. Kanayama, J. Cryst. Growth 237 (2002) 1495.

[15] A. Zaoui, Mat. Sci. Eng. B 103 (2003) 258. 
[16] A. Ferreira da Silva, A. N. Souza Dantas, J. S. de Almeida, R. Ahuja, C. Perssone, J. Cryst. Grow. 281 (2005) 151.

[17] L. Shi, Y. Duan, L. Qin, Comput. Mater. Sci. 50 (2010) 203

[18] L.-W. Shi, Y.-F. Duan, L.-X. Qin, Chin.Phys.Lett. 27 (2010) 0880505.

[19] L.-W. Shi, Y.-F. Duan. Y.-F. Yang, L.-X. Tang, Chin. Phys. Lett. 28 (2011) 100503.

[20] N. Souza Dantas, J. S. de Almeida, R. Ahuja, C. Persson, A. Ferreira da Silva, Appl. Phys. Lett 92 (2008) 121914.

[21] L. Shi, Y. Duan, X. Yang, G. Tang, L. Qin, L. Qiu, Matter. Sci. Semicond. Process. 15 (2012) 499.

[22] N. Saidi-Houat, A. Zaoui, A. Belabbes, M. Ferhat, Mat. Sci. Eng. B 162 (2009) 26.

[23] F. Tran, P. Blaha, Phys. Rev. Lett 102 (2009) 226401.

[24] Y.-S. Kim, M. Marsman, G. Kresse. F. Tran, P. Blaha, Phys. Rev. B 82 (2010) 205212.

[25] X. Gonze et al., Comput. Mater. Sci. 25 (2002) 478.

[26] X. Gonze et al., Comput. Phys. Commun. 180 (2009) 2582.

[27] A. R. Tackett, N. A. W. Holzwarth, G. E. Matthews, Comput. Phys. Commun. 135 (2001) 329.

[28] J. P. Perdew, Y. Wang, Phys. Rev. B 45 (1992) 13244.

[29] M. Oliveira, F. Nogueira, Comput. Phys. Comm. 178 (2008) 524.

[30] P. Blaha, K. Schwarz, P. Sorantin, S. B. Trickey, Comp. Phys. Commun. 59 (1990) 399.

[31] P. Scharoch, M. J. Winiarski, M. P. Polak, Comput. Mater. Sci. 81 (2014) 358.

[32] S. Q. Wang, H. Q. Ye, J. Phys.: Condens. Matter 14 (2002) 9579. 
Table 1: Lattice parameter $a$, band-gap $\mathrm{E}_{g}$, split-off energy $\left(\Delta_{S O}\right)$ of zinc blende $\mathrm{InN}$ and TIN

\begin{tabular}{llll} 
& $a(\AA)$ & $\mathrm{E}_{g}(\mathrm{eV})$ & $\Delta_{S O}(\mathrm{eV})$ \\
\hline InN: & & & \\
LDA & 4.948 & 0.00 & 0.393 \\
MBJLDA & - & 0.69 & 0.033 \\
ref [31] & 4.970 & 0.64 & - \\
ref [32] & 4.801 & 0.75 & - \\
ref [33] & - & - & 0.013 \\
TIN: & & & \\
LDA & 5.136 & 0.03 & 2.083 \\
MBJLDA & - & 0.11 & 1.397 \\
ref[16] & 5.139 & - & - \\
ref[22] & 5.133 & - & 2.000
\end{tabular}

[33] M. Cardona, N.E. Christensen, Solid State Commun. 116 (2000) 421.

[34] F. Virot, R. Hayn, M. Richter, J. van den Brink, Phys. Rev. Lett. 106 (2011) 236806.

[35] L. Fu, C. L. Kane, Physical Review B 76 (2007) 045302.

[36] W. Feng, W. Zhu, H. H. Weitering, G. M. Stocks, Y. Yao, D. Xiao, Phys. Rev. B 85 (2012) 195114.

[37] J. Iveland, L, Martinelli, J. Peretti, J.S. Speck, and C. Weisbuch, Phys. Rev. Lett. 110 (2013) 177406.

[38] Z. Batool, K. Hild, T. J. C. Hosea, X. Lu, T. Tiedje, S. J. Sweeney, J. Appl. Phys. 111 (2012) 113108.

[39] M. Usman, C. A. Broderick, Z. Batool, K. Hild, T. J. C. Hosea, S. J. Sweeney, E. P. O'Reilly, Phys. Rev. B 87 (2013) 115104. 

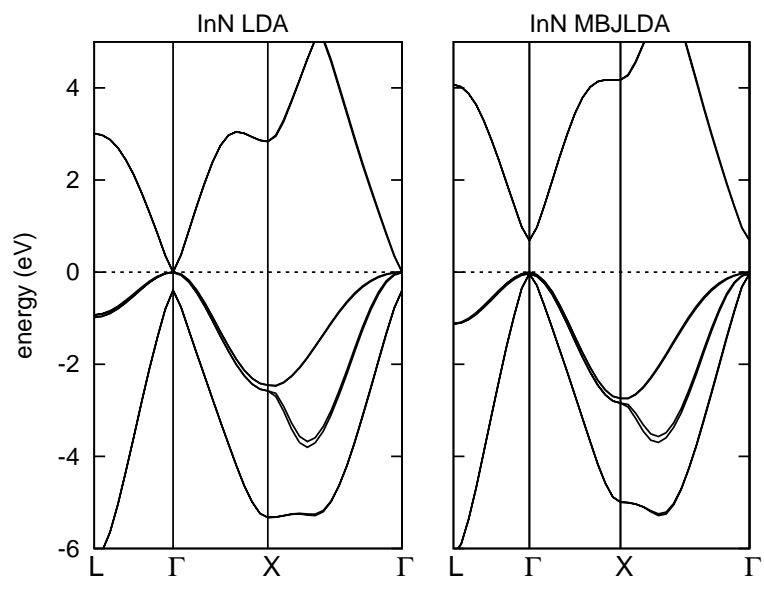

Figure 1: Band structure of zinc blende InN, calculated within LDA and MBJLDA.
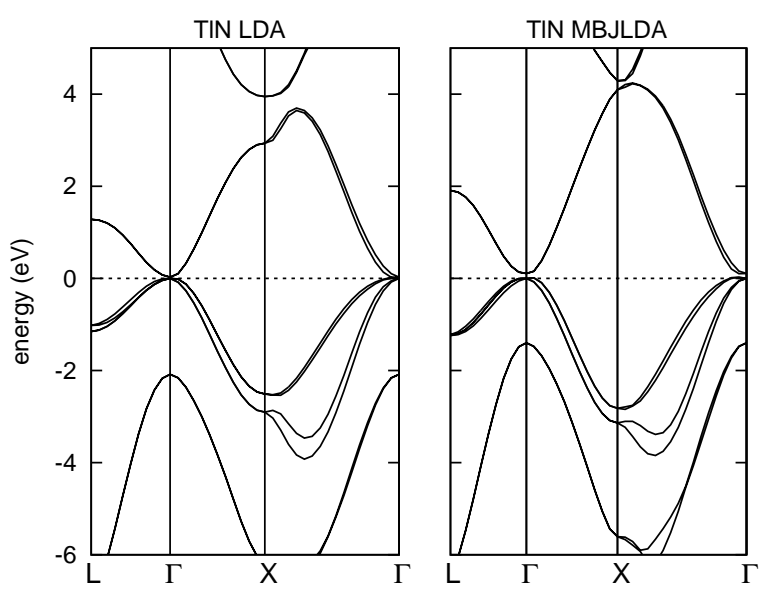

Figure 2: Band structure of zinc blende TIN, calculated within LDA and MBJLDA. 


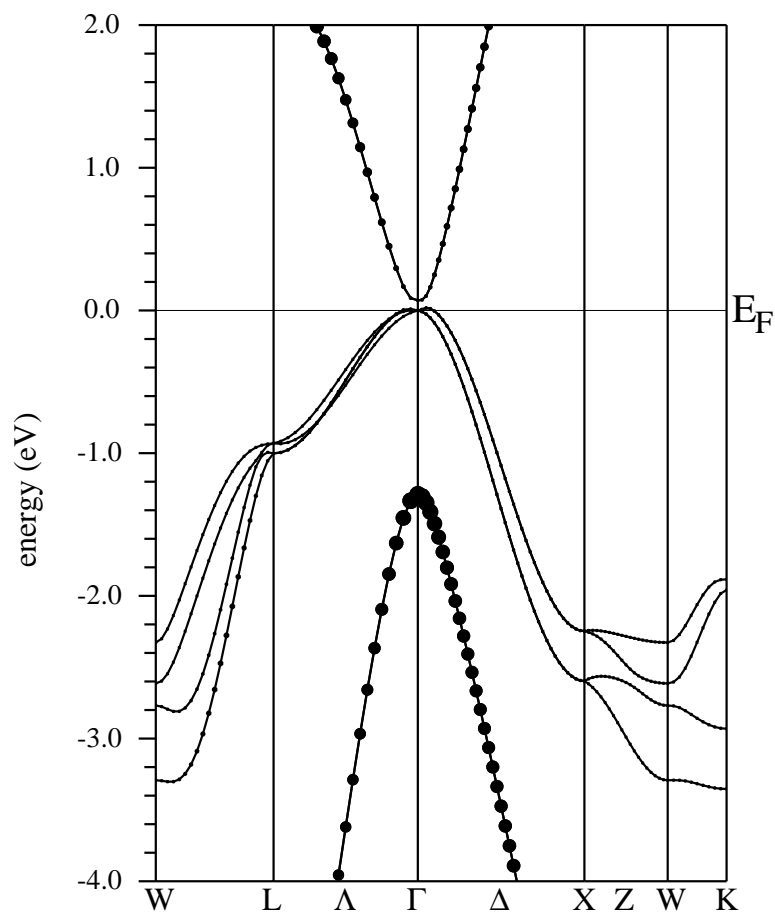

Figure 3: Orbital band characters (fat bands) for s-electrons of $\mathrm{Tl}$ atom in zinc blende TIN indicating the s-type character of split-off band (the minimum of the conduction band is dominated by p-electrons of $\mathrm{N}$ atom).

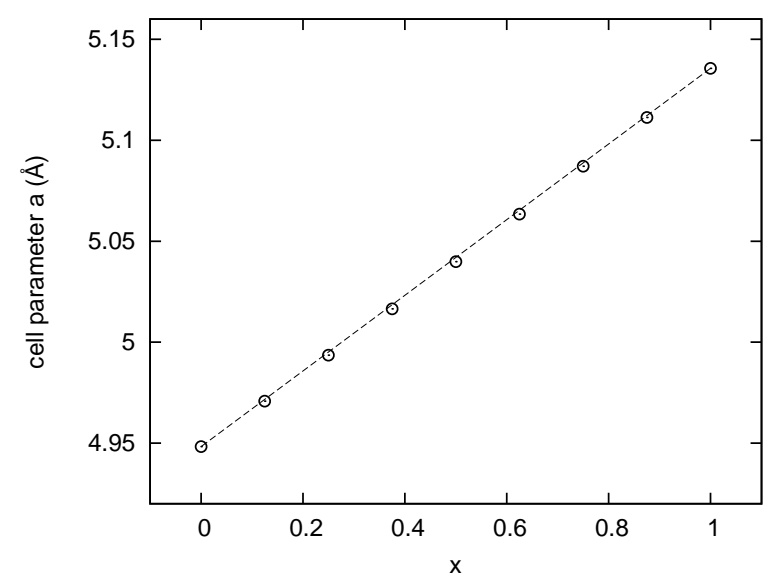

Figure 4: LDA calculated lattice parameters of zinc blende $\mathrm{Tl}_{x} \operatorname{In}_{1-x} \mathrm{~N}$ alloy as a function of thalium content. 


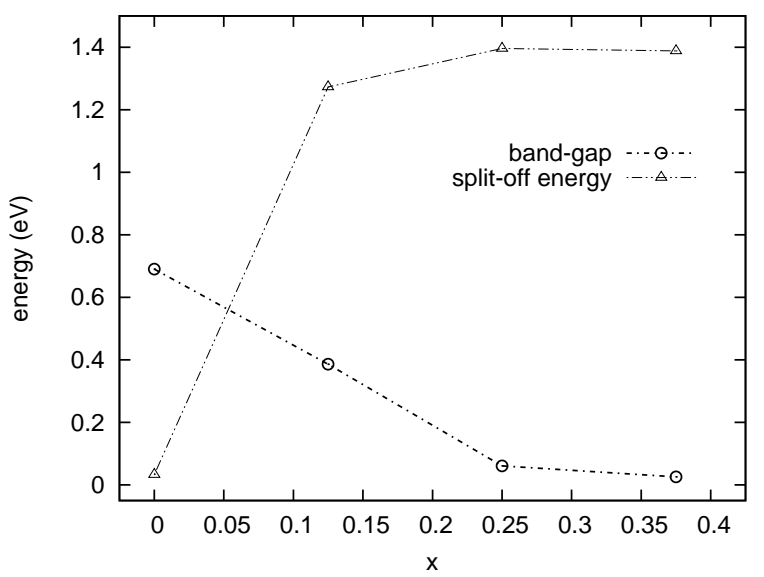

Figure 5: MBJLDA calculated band-gap and split-off energy for $\mathrm{Tl}_{x} \operatorname{In}_{1-x} \mathrm{~N}$ alloy. 